\title{
AN OVERVIEW OF THE USE OF BIOMETRIC TECHNIQUES IN SMART CITIES
}

\author{
Elham Farazdaghi, Mojtaba Eslahi, Rani El Meouche \\ Institut de Recherche en Constructibilité (IRC), ESTP Paris, 94230 Cachan, France \\ (efarazdaghi, meslahi, relmeouche)@estp-paris.eu
}

KEYWORDS: Smart City, Smart Building, Biometrics Systems, Face Recognition, Security, Smart Healthcare, Smart advertisement.

\begin{abstract}
:
The human desire to live in an urban area increases every day. However, citizens' expectation of urban life is very different compared to the past. It is, thus, essential to satisfy their requirements and ensure their safety within their cities. As a result, there is a huge trend in the implementation of smart cities around the world. A smart city is a solution to improve the quality of life of the citizens, and governing the city in an efficient and systematic. Besides, significant advances have been raised in biometrics technologies, which have made many aspects of urban life easier, more efficient, and more secure. Accordingly, to be compliant with the demands of a smart city in the future, biometrics-based technologies will certainly play a significant role from now on. Thus, it is necessary to list the different biometrics methods that could be used in smart cities and to review the variety of applications for each method. In this article, we have listed the potential biometrics systems that can be employed in smart cities, such as facial recognition, age estimation, gender detection, facial expression detection and sentiment recognition, and gait recognition. We also have listed different applications imagined for each biometrics system such as their application in identification systems and security, smart healthcare, smart advertising, education, and high-risk lifestyle behaviours prevention. We believe that this work can help to better use of these methods, improve their technical quality, and also employing them in the advance and more effective ways.
\end{abstract}

\section{INTRODUCTION}

The tendency to urbanization is increasing every day, to the extent that today more than $50 \%$ of the world's population lives in cities. With the expansion of urbanization and the advancement of technology, it is impractical to live in, or traditionally govern the cities. As a result, the design of smart cities is a trending topic of the day. Smart people, smart governance, smart environment, and smart living are some of the attributes of a smart city. However, citizens are surely in the center of smart cities, and in the favoured smart cities services and policies are developed with the citizens in focus. Thus, it is essential to satisfy the needs of the inhabitants and to ensure their safety within their cities. On the other side of today's developed world, there are biometrics systems. A biometric system employs the measurement and statistical analysis of human's unique physical and behavioural characteristics (Jain et al., 2011). In recent years, large cities are starting to use biometric techniques to improve the living conditions of their citizens. Indeed, smart cities are connected cities using all kinds of technologies to improve the daily life of the inhabitants. There are different types of biometrics methods including facial recognition, fingerprints, finger geometry, iris recognition, vein recognition, retina scanning, voice recognition, DNA matching, digital signatures, etc. Each of these technics separately or in combination with other methods have applications in smart cities (Zhang, 2013).

This study intends to provide an overview of the applications of biometrics techniques in the development of smart cities. It includes a wide range of biometric technologies that could be employed in smart cities; such as facial recognition, age estimation, gender detection, facial ageing modelling, gait recognition, emotion, expression, and sentiment recognition, and voice recognition. undoubtedly, discussion of all the applications of the mentioned biometrical technics will be hardly possible, but in this work, we intend to examine these techniques in different applications such as in identification and security, smart healthcare, smart marketing, education, and high-risk lifestyle behaviours prevention specially for the young generation. We hope that in the future, new and attractive ideas will be generated from completing or combining these applications. Perusing the positive points and the limitations of each technique can certainly lead to overcome disadvantages and provide more efficient performance in the future and the emergence of new and practical ideas. These classified techniques and applications can be used to provide an appropriate view for smart city decision-makers and planners.

The rest of this paper is organized as follows: In Section 2 we have explained the functional architecture of a biometrics system. In Section 3 we have discussed the different biometrics methods that can be employed in smart cities with their possible applications. The discussions and conclusions are given in Section 3.

\section{THE FUNCTIONAL ARCHITECTURE OF A BIOMETRICS SYSTEM}

A biometric system is generally composed of two main modes consisting of the learning mode, also called the enrolment mode, and the recognition mode, including the verification or identification. The first mode involves recording the physical or behavioural characteristics of an individual in a database in the form of a biometrics model, also called template or signature. The second mode consists of testing the same characteristics and comparing them with the biometrics templates stored in the database. If the tested data matches an enrolled biometric template, the individual is considered as recognized. A generic biometric system can be viewed as a four-module process including:

1. Capture module: A suitable biometric reader or scanner is required to acquire biometric data from an individual.

2. Pre-processing and feature extraction module: The quality of the biometric data acquired by the biometric sensor is first evaluated to determine its suitability for further processing.

3. Matching module: The extracted feature vector is compared with the templates stored in the database to generate match scores. 
4. Database management module: The database acts as a repository of biometric data.

\subsection{Recognition And Decision Threshold}

In the verification case, a user has to present his biometric trait and proclaim his identity to the biometric system, the latter computes a similarity (or dissimilarity) score/distance (between 0 and 1) between the feature vector extracted from the presented trait and the feature vector of the same user registered in the database (enrolled vector). The closer the similarity score is to 0 , the more confident the system is that the two patterns come from the same individual, in this case, the individual is named: genuine/authentic. The closer the score is to 1 , the more the system is sure that the models come from two different individuals, in this case, the proclaimed individual is named: impostor. The decision is made by choosing a decision threshold $\theta$; a similarity score less than or equal to $\theta$ indicates that the two compared models belong to the same individual, and a score greater than $\theta$ indicates that the two compared models come from two different individuals.

\section{BIOMETRICS METHODS COULD BE EMPLOYED IN SMART CITIES}

\subsection{Facial Recognition}

Since the beginning of 1970 , face recognition has received so much attention from researchers and companies, as it has great potential in many commercial and governmental applications. In the face recognition systems, the system is trained using an enrolment part in which $\mathrm{N}$ images are captured from the detected face and stored in a database with a name. Then, every time that a face is detected, the system finds a face, searches in the database, and matches them using a similarity threshold to minimize the chance of false positives. There are different techniques and algorithms for facial recognition (Parmar and Mehta, 2014), and more accurate and reliable methods are being offered every day, which with the combination of good quality cameras and better processing powers their performances are noteworthy. There are different approaches for face recognition, such as statistical-based approaches, appearance-based holistic approaches, feature-based graph matching approaches, and artificial intelligence-based approaches (Beham and Roomi, 2013). The discussion of these techniques is beyond the scope of this article; thus, we will discuss the applications of face recognition in developing smart cities.

3.1.1 Identification: By far the most common application of facial recognition is identification. Face recognition systems identify people by their facial images. There are many innovative applications for identification, such as unlocking smartphones, computer systems, access controls etc. The applications of face identification are far beyond imagination. So that it can cover different aspects of urban life from inside the house to the sky. Some of these applications are: smart TVs, groceries payment by face, prevent shoplifting and violent crimes in retail shops, ATM withdrawals, catch the trains, pass through airport security gates without showing passports and waste the time, identify suspects and dead bodies by forensics and law enforcement, attendance systems, and any place where needs access control with any level of security (Jafri and Arabnia, 2009). In general, human-based identification methods require great physical efforts, also employees can make errors in overloaded situations or by some distractions.

Automating this process can improve the security of sensitive areas and reduce the needs of the labour force. It can be said, facial recognition applications promise a vast range of benefits and conveniences, such as security and speed (Center, 1998).

3.1.2 Health Care: one of the important characteristics of a successful smart city is its healthcare facilities. Medical applications of facial recognition may provide the basis for improved diagnosis and could be the end to ethical discrimination. For instance, in many emergency cases, time is very short to help the patient. Sometimes the person is not able to talk, his ID card is not available, and contact with his relatives is not possible for identifying him and knowing his medical backgrounds such as medications, and MRI. In such cases, face recognition comes to the aid of the medical team and provides the patient's medical records. Moreover, facial recognition systems can help health centres tackle patient fraud. It can also be used in detecting and identifying people with a history of drug addiction.

3.1.3 Education: Another application of facial recognition is in schools and educational centres. These systems can be employed in attendance monitoring systems, which has the potential to end the gaps and skips spatially in classes with a large number of students. This kind of systems can help disciplinary practices in schools. Moreover, facial recognition can be used in virtual learning for controlling access to online educational contents. Furthermore, in the computer-based exams facial identification, apart from identifying the students can help to control the continued presence of them during the whole examination period.

3.1.4 Profile Setting: In general, people spend most of their time in the building, such as at home, in the office, and in shopping centres. Therefore, smart buildings are an important part of smart cities. Biometric systems are being used in many parts of the building. For instance, one interesting use of face recognition systems is for profile setting in the different part of the building. It says one can set his own profile with his desired light, temperature, and the level of ventilation of the room. This can reduce the energy consumptions in the smart buildings, provide thermal and visual comfort for the habitants, and save time. Profile setting has also other applications. For example, in their computers, people can set a specific font or settings under their profiles, or in the audio-visuals systems, people can use this technique for the automatic choice of their preferred music and TV channel, and automatic volume levelling of the systems.

\subsection{Age Estimation and Gender Detection}

Facial age estimation can be described as the estimation of the age of an individual based on the biometrics facial features based on facial images. The pioneers of age classification are (Kwon and Lobo, 1999) that categorize the face images into only three different age groups: babies, young adults, and seniors, using the craniofacial development theory and skin analysis. Since then, many efforts have been made in this field (Horng et al., 2001) (Izadpanahi and Toygar, 2012). It could be said that in recent years, very high accuracy results in estimating the age have been achieved.

3.2.1 Smart advertising: Advertisements are a way to improve the visibility of businesses in the world. Individual marketing is a new way of attracting customers in smart cities. This kind of marketing targets special members of society such as a specific gender or age group.

Using age estimation and gender detection technics in digital advertisement boards in the cities, the faces that are attracted by a special part of the ad are detected, and the data for gender-wise 
and age-wise ads are collected by counting the amount of audience and estimation of their age categories. In the next level, special algorithms are employed to choose an advertisement from databases and make an advertisement loop using the detected gender and estimated age group (Fonseka and Naleer, 2019) (Sumer et al., 2018).

3.2.2 Smart Health Care: There are some legal age restrictions for drinking, smoking, and access to sexual or violent content in many countries. The legal drinking and smoking age is the minimum age at that a person can legally purchase alcoholic beverages or tobacco products. The most known reason for the legal drinking age law is the alcohol impact on the adolescent brain. Since the brain is still maturing, alcohol can have a harmful effect on long-term memory and thinking. It also can have negative effects on hormone balance and the liver (MacMahon, 1987) ("Underage Drinking | National Institute on Alcohol Abuse and Alcoholism). The control on the sale of these products is very challenging because in many cases sellers circumvent the law or cigarette vending machines are an alternative option for non-controlling purchases. Therefore, using the age estimation systems embedded within cigarette vending machines and stores can greatly help to control the sale of alcohol, cigarettes, and any other age-restricted product to protect the young generation from any physical or mental problems caused by them.

3.2.3 Content censorship: The intelligent vision systems can be integrated based on biometrics systems, which together with a camera and a TV generate an intelligent vision system for content censorship. The principle is that the television system adapts the video stream based on its audience age group. This kind of system censor, mask or adapt the scene inappropriate for the younger, or elderly public (if there are violent scenes not suitable for older people and people with heart problems), regulates the time spent by children watching $\mathrm{TV}$, and propose adapted programs to viewers. Audience recognition can be implemented in two modes: supervised mode, in which a special user provides the required age information to the system. It means samples, names, and other related information are fed to the system, age is specified manually by admin, and the system uses facial recognition algorithms. Non-supervised mode, in which the system uses biometric analysis to determine the age of every person in front of the TV. Therefore, age is estimated by the system using automatic age estimation algorithms.

\subsection{Facial Ageing Modelling}

Ageing is a definite phenomenon that happens for every single person around the world and there no way to stop it. The ageing pattern of each individual is different from the others. The facial ageing process in each person could be linked to numerous innate factors such as ethnicity, genetics, gender, and many external causes such as a person's lifestyle habits, living and work environment, and many other factors that can change the ageing process. Instead, even though ageing is generally distinct from one person to another, individuals in the same age group have certain facial similarities. Study of these similarities and differences can help scientists to a better understanding of this phenomenon. Automatic facial ageing modelling has been noticed in recent years. "Facial ageing modelling is the illustration of an individual's facial appearance in the bygone or the forthcoming years, by simulating the alterations caused by facial ageing trajectory and by using the biometric facial features" (Farazdaghi, 2017). Face ageing models can be divided into two groups: "Predictive Facial Ageing Models" which model the ageing process and show the face in the coming years or decades (Burson and Schneider, 1981)(Ramanathan and Chellappa, 2008), and "Reconstructive Facial Ageing Models" that intend to rejuvenate the facial images from old adult to young adult (Bastanfard et al., 2004), or from adult to the child (Farazdaghi and Nait-ali, 2017) (Heravi et al., 2016). We also can add the destructive effects of bad behavioural habits to the predictive facial ageing models in order to show people specially young people the harmful results of these habits on the face (Farazdaghi and Nait-Ali, 2016).

Many applications could be imagined for a trusty face ageing model such as its application in anthropology, finding missing people, forensics, a criminal investigation, modelling suspects' faces, automatic updating of biometric systems, age normalization in face recognition systems, cosmetic surgery, the facial transplant planning, visual arts, movie special effects in the film industry, and high-risk lifestyle behaviours prevention.
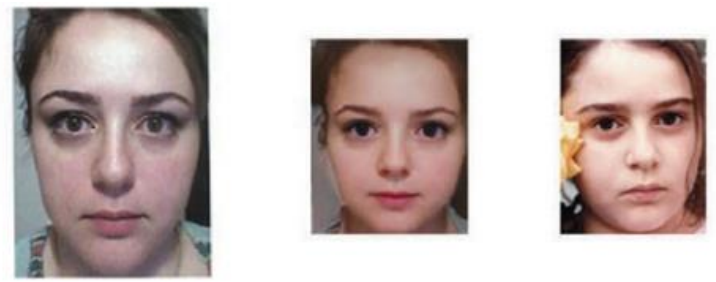

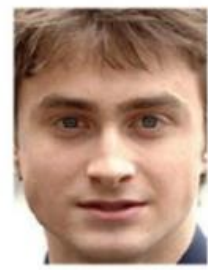

(a)

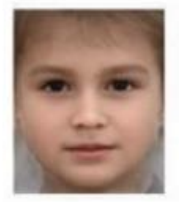

(b)

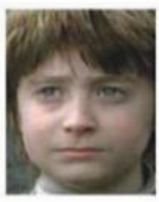

(c)
Figure 1. Results of the reconstructive facial ageing model (a) Original image - young adult. (b) Result (c) Reference image in approximately the same age as the result, for comparison. (Farazdaghi and Nait-ali, 2017) (Nait-Ali, 2019).

3.3.1 Finding Missing People: At first glance, a reconstructive facial ageing model that generates the facial image of the individual in his childhood may seem like a fantasy, and its application may not be evident. However, one of the most interesting utilization of this model can help to find the missing people, specially those who have disappeared as a child, and the only sign left of them is a photo of their childhood. Thus, the system can use the facial image taken by surveillance systems to model the childhood face. The comparison with the available reference photos from the person's childhood may give important information to the police and investigators (Figure 1).

3.3.2 High-Risk Lifestyle Behaviours Prevention: Facial appearance is very important for people and they always want to keep it younger and more beautiful. Bad lifestyle habits, such as drug abuse, alcohol consumption, smoking and extreme sun exposure can change the facial appearance and ageing process. One of the risky lifestyle habits is drug addiction. Many people are trapped in drug addiction and their number is increasing every day. Though, the media talks a lot about drug abuse and its effects on health and appearance, unfortunately, many teenagers begin to use drugs every day. All substances such as heroin, cocaine, amphetamines, marijuana, etc. have serious negative mental and physical effects. Some of these physical effects could be the rash of red bumps over the skin, appearance of wrinkles and 
premature ageing, serious dental and oral problems, loss of appetite, and as a result weight loss (Shekarchizadeh et al., 2013).

Integration of the effect of bad lifestyle habits in the facial ageing models, in addition to offering more realistic and personalized results, can demonstrate the future of a young face in case of choosing the high-risk lifestyle and it can be an alarm for people specially teenagers to prevent and even advance stopping the addiction to the fatal substances (Figure 2). This kind of ageing model systems can be installed in schools, bars, clubs, and other urban areas that young people spend their time.

\subsection{Expression, Emotion and Sentiment Recognition}

Humans are highly skilled at expressing emotions using facial expressions. Facial expression can reflect emotions, mental activities, physiological signs, and social interactions. Therefore, automatic recognition of facial expressions can have a lot of applications in modern life (Du and Martinez, 2015).

3.4.1 Health care: One of the important factors of a desirable city to live in is the mental health of its citizens. Early diagnosis of depression and detection of the level of anger in people can prevent many incidents. Human facial expressions alter depending on the state of health, mental state or even the level of anger. Therefore, a facial expression recognition system can be useful in a healthcare setting and understanding the sentiment and emotion state of the patients. Various techniques have been proposed to recognize and classify individual emotions. The three main technics are speech emotion recognition which identifies the emotional state of the person by analysing the voice of the individual, emotion recognition from a facial expression which recognizes the emotion of an individual from the facial muscle, eyes, eyebrows movements, and facial texture, and emotion recognition using biological signals such as EEG or ECG (Altameem and Altameem, 2020).

The emotion recognition systems can be used in online psychological counselling sessions or annual medical check-ups in order to early diagnosis of mental health problems and depressions. The importance of this kind of systems has become more apparent with the Covid-19 pandemic, and it may be necessary to do more research and work to improve this kind of systems for the mental support of the citizen in difficult and critical moments. It can also be employed to diagnose the pain in people with speech disabilities or mobility impairments.

3.4.2 Smart Marketing: Another application of emotion and expression recognition systems can be in modern marketing. Human facial expression can show the level of satisfaction, curiosity, or excitement of the individual by seeing a product. Therefore, these systems can be positioned in the store windows or near the shelves to recognize facial emotions and record people's level of interest and attention for different products.

\subsection{Gait Recognition}

Gait recognition is a behavioural biometrics technic that means the automatic recognition of people by the way they walk. Studies show that a person's gait is certainly unique. Since gait recognition does not require advanced instruments and highresolution images, the data acquisition of an individual's gait can be easily done, and public areas gait recognition does not require subject assist, so, it can be used in many different applications such as monitoring and access control of high-security areas, criminal investigations, and automated surveillance, and home automation (Boyd and Little, 2005). In addition to its applications in security, other applications for gait analysis can be imagined (Makihara et al., 2015).

3.5.1 Smart Healthcare: One of the interesting applications of gait analysis is gait-related pathology. Gait analysis can help the healthcare system in two different ways. First, it can help to diagnose gait-related disorders. Then to monitor the process of the treatment and rehabilitation such as in cerebral palsy children or any patient with a walking disorder. This kind of healthcare systems can compensate for clinical deficiencies and give consistent diagnoses and assessments of treatments to the patients (Boyd and Little, 2005). Gait recognition can also be used in the home security systems for elderly individuals, to track their walking and detect if they fall and send alerts to the emergency numbers.
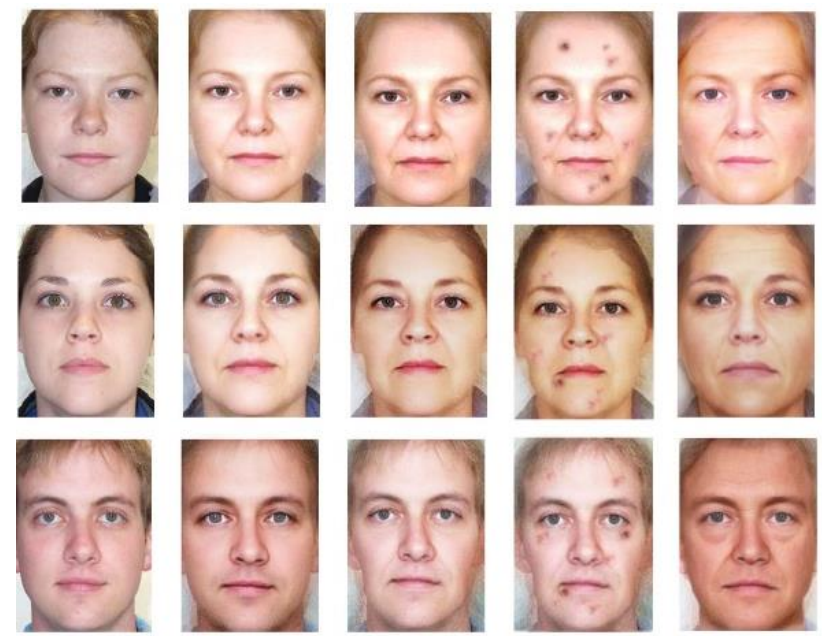

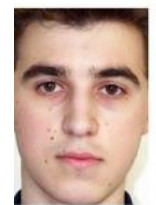

(a)

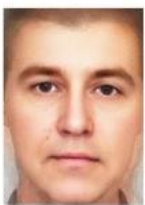

(b)

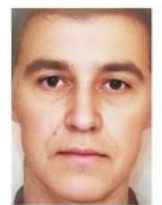

(c)

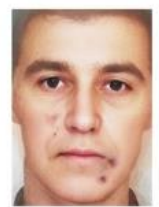

(d)

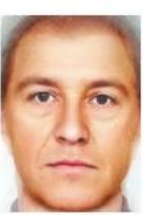

(e)
Figure 2. Results of applying predictive models to compare. (a) Input image (b) Output of applying natural ageing model (c) Outputs of applying the behavioural model for Methamphetamine addicts (d) Outputs of applying the behavioural model for Methamphetamine addicts if consumption continues for a longer time. (e) Results of applying the behavioural model for the sun-seeking people - The output age range for all the results is 41-50 (Farazdaghi and Nait-ali, 2017).

\section{CONCLUSION AND PERSPECTIVES}

In this article, we have presented an overview of the different biometrics technics applications that can be utilized in smart cities design and development. Some of these techniques such as face recognition have been already had various applications in public and private sectors of cities for many years, and the use of some is recent. Anyhow, categorizing these methods, and the close study of their diverse attributes and applications can be a vision for the construction of the desired and ideal cities in the future.

We have considered five different practical and useful biometrics systems categories including facial recognition, age estimation and gender detection, facial ageing modelling, expression, emotion and sentiment recognition, and gait recognition. Then, for each of them, we have mentioned the real and practical 
applications in different fields such as security, investigation, healthcare, education, marketing and advertisement, and highrisk prevention.

Of course, there is still a lot of place to work, and there are many other useful biometrics technics, and for each, a lot of interesting applications that we can consider in the feature work. Giving the advantages and disadvantages of each application and system could also be valuable.

\section{REFERENCES}

Altameem, T., Altameem, A., 2020. Facial expression recognition using human machine interaction and multi-modal visualization analysis for healthcare applications. Image and Vision Computing 103, 104044.

Bastanfard, A., Bastanfard, O., Takahashi, H., Nakajima, M., 2004. Toward anthropometrics simulation of face rejuvenation and skin cosmetic. Comp. Anim. Virtual Worlds 15, 347-352.

Beham, M.P., Roomi, S.M.M., 2013. A review of face recognition methods. Int. J. Patt. Recogn. Artif. Intell. 27, 1356005 .

Boyd, J.E., Little, J.J., 2005. Biometric Gait Recognition, in: Tistarelli, M., Bigun, J., Grosso, E. (Eds.), Advanced Studies in Biometrics: Summer School on Biometrics, Alghero, Italy, June 2-6, 2003. Revised Selected Lectures and Papers, Lecture Notes in Computer Science. Springer, Berlin, Heidelberg, pp. 19-42.

Burson, N., Schneider, T.D., 1981. Method and Apparatus for Producing an Image of a Person's Face at a Different Age.

Center, J.L., 1998. Practical Application of Facial Recognition: Automated Facial Recognition Access Control System, in: Wechsler, H., Phillips, P.J., Bruce, V., Soulié, F.F., Huang, T.S. (Eds.), Face Recognition: From Theory to Applications, NATO ASI Series. Springer, Berlin, Heidelberg, pp. 402-411.

Farazdaghi, E., 2017. Facial ageing and rejuvenation modeling including lifestyle behaviours, using biometrics-based approaches (Theses). Université Paris-Est.

Farazdaghi, E., Nait-ali, A., 2017. Backward Face Ageing Model (B-FAM) for Digital Face Image Rejuvenation. IET Biometrics.

Farazdaghi, E., Nait-Ali, A., 2016. Face aging predictive model due to methamphetamine addiction, in: 2016 International Conference on Bio-Engineering for Smart Technologies (BioSMART). Presented at the 2016 International Conference on Bio-engineering for Smart Technologies (BioSMART), pp. 1-4.

Flament, F., Bazin, R., Laquieze, S., Rubert, V., Simonpietri, E., Piot, B., 2013. Effect of the sun on visible clinical signs of aging in Caucasian skin. Clin Cosmet Investig Dermatol 6, 221-232.

Fonseka, G.W.I.R., Naleer, H.M.M., 2019. Face-recognition billboard display to target advertisement with gender and age recognizing.

Heravi, F.M.Z., Eslahi, M., Farazdaghi, E., Nait-Ali, A., 2016. A Morphable Model to simulate rejuvenation trajectory of 3D face images: Preliminary results, in: 2016 International Conference on Bio-Engineering for Smart Technologies (BioSMART). Presented at the 2016 International Conference on Bioengineering for Smart Technologies (BioSMART), pp. 1-4.
Horng, W.-B., Lee, C.-P., Chen, C.-W., 2001. Classification of age groups based on facial features. 淡江理工學刊 4, 183-192.

Izadpanahi, S., Toygar, Ö., 2012. Geometric feature based age classification using facial images, in: IET Conference on Image Processing (IPR 2012). Presented at the IET Conference on Image Processing (IPR 2012), pp. 1-5.

Jafri, R., Arabnia, H.R., 2009. A Survey of Face Recognition Techniques. Journal of Information Processing Systems 5, 4168.

Jain, A.K., Ross, A.A., Nandakumar, K., 2011. Introduction to Biometrics. Springer Science \& Business Media.

Kwon, Y.H., Lobo, N. da V., 1999. Age Classification from Facial Images. Computer Vision and Image Understanding 74, $1-21$.

MacMahon, S., 1987. Alcohol consumption and hypertension. Hypertension 9, 111-121.

Makihara, Y., Matovski, D.S., Nixon, M.S., Carter, J.N., Yagi, Y., 2015. Gait Recognition: Databases, Representations, and Applications, in: Wiley Encyclopedia of Electrical and Electronics Engineering. American Cancer Society, pp. 1-15. https://doi.org/10.1002/047134608X.W8261

Nait-Ali, A. (Ed.), 2019. Biometrics under Biomedical Considerations, Series in BioEngineering. Springer Singapore.

Parmar, D.N., Mehta, B.B., 2014. Face Recognition Methods \& Applications. arXiv:1403.0485 [cs].

Ramanathan, N., Chellappa, R., 2008. Modeling shape and textural variations in aging faces. IEEE, pp. 1-8.

Shekarchizadeh, H., Kahmi, M.R., Mohebbi, S.Z., Ekhtiari, H., Virtanen, J.I., 2013. Oral Health of Drug Abusers: A Review of Health Effects and Care. Iran J Public Health 42, 929-940.

Sumer, E., Sumer, S.I., Ogul, H., 2018. A Novel Computer Vision-Based Advertisement System for Individual Marketing.

Underage Drinking | National Institute on Alcohol Abuse and Alcoholism (NIAAA) [WWW Document], n.d. URL https://www.niaaa.nih.gov/publications/brochures-and-factsheets/underage-drinking (accessed 3.14.21).

Yin, R., Chen, Q., Hamblin, M.R., 2015. Skin Photoaging. Morgan \& Claypool Publishers.

Zhang, D.D., 2013. Automated Biometrics: Technologies and Systems. Springer Science \& Business Media. 